\title{
Multitone-Hopping CDMA Using Feedback-Controlled Hopping Pattern for Decentralized Multiple Access
}

\author{
Kazuki CHIBA $^{\dagger \text { a) }}$, Student Member and Masanori HAMAMURA ${ }^{\dagger \dagger}$, Member
}

\begin{abstract}
SUMMARY We propose multitone-hopping code-division multiple access (MH-CDMA) using a feedback-controlled hopping pattern (FCHP) (FCHP/MH-CDMA). In the FCHP/MH-CDMA, part of the filter coefficients of an adaptive finite-duration impulse response (FIR) filter receiver are fed back to a transmitter, in which they are used as an updated hopping pattern. Each chip of the updated hopping pattern consists of plural tones. As a result, it is shown that the FCHP/MH-CDMA provides us with an excellent asynchronous, decentralized multiple-access performance over time-invariant multipath channels.

key words: CDMA, decentralized, asynchronous, multipath, feedback, multitone
\end{abstract}

\section{Introduction}

The performance of asynchronous wireless communication systems is deteriorated by intersymbol interference (ISI) due to multipath and multiple-access interference (MAI) [1][4]. It is well-known that code-division multiple access (CDMA) can suppress ISI and MAI, resulting in high signalto-interference plus noise ratio (SINR). The CDMA can be categorized into direct-sequence CDMA (DS-CDMA) or frequency-hopping CDMA (FH-CDMA).

The DS-CDMA can suppress ISI and MAI through the process of despreading at the receiver. In addition, the DSCDMA can further improve the SINR by applying a combining method such as RAKE and Pre-RAKE [1], [4], [5]. Another method for ISI and MAI suppression in DS-CDMA is to apply adaptive filters [6]-[9], which can reduce ISI and MAI without any explicit knowledge of channel-state information.

Similarly, the FH-CDMA can suppress ISI and MAI by choosing an appropriate hopping pattern. A hybrid of DS- and FH-CDMA can also be applied to suppress ISI and MAI.

To greatly reduce ISI and MAI, feedback-based systems have been studied. For uplink of the asynchronous DS-CDMA, a method in which a base station (BS) employs an adaptive filter at the receiver to produce an analog pseudo-noise (PN) sequence, which is assigned to a new user, was proposed [9]. The analog PN sequences can be orthogonal to each other under arbitrary asynchronous con-

\footnotetext{
Manuscript received January 16, 2008.

Manuscript revised May 12, 2008.

$\dagger$ The author is with the Department of Engineering, Kochi University of Technology, Kami-shi, 782-8502 Japan.

${ }^{\dagger}$ The author is with the Graduate School of Engineering, Kochi University of Technology, Kami-shi, 782-8502 Japan.

a)E-mail: chiba@m.ieice.org

DOI: 10.1093/ietfec/e91-a.12.3723
}

ditions. For a synchronous DS-CDMA, an iterative construction method that produces signature sequences using a minimum mean-squared error (MMSE) filter was proposed [10], which has been shown to produce a set of Welch bound equality (WBE) sequences [11], [12], which minimize the total squared cross-correlation. This method employs an MMSE filter whose size is identical to the length of the signature sequence. In contrast, we have proposed another DS-CDMA using feedback-controlled spreading sequences (FCSS/DS-CDMA) [13], [14]. In the FCSS/DS-CDMA, the receiver employs an adaptive filter whose size is larger than that of the signature sequence, and returns part of the filter coefficients to the transmitter. It has been shown that this method provides us with superior performance in terms of bit-error rate (BER) over time-invariant multipath channels to that of the method in [10].

In this paper, we propose multitone-hopping $(\mathrm{MH})$ CDMA using a feedback-controlled hopping pattern (FCHP) (FCHP/MH-CDMA), which combines the FHCDMA with the FCSS/DS-CDMA, to enhance the improvement of SINR. Each receiver of the FCHP/MH-CDMA is composed of a time-frequency, 2-dimensional, adaptive finite-duration impulse response (FIR) filter, whose size is larger than that of the hopping pattern. The receiver returns part of the filter coefficients to the transmitter. In this paper, we mainly focus on the decentralized multiple-access performance of the proposed CDMA over time-invariant multipath channels. Results are shown in comparison with those for several conventional CDMA systems.

\section{System Model}

\subsection{Transmitter}

We assume a decentralized multiple access such as that in wireless ad-hoc networks where no BS exists and all transmitters asynchronously transmit their signals to the corresponding receivers, as illustrated in Fig. $1^{*}$. Therefore, the received signal for decentralized multiple access can be modeled as a sum of $K$ signals that are independently transmitted through different channels, as shown in Fig. 2, where $\mathrm{Tx}_{k}$ and $\mathrm{Rx}_{k}(k=1,2, \cdots, K)$ are the transmitter and receiver for the $k$ th signal, respectively. The transmitter and

${ }^{*}$ Although we assume asynchronous multiple access, synchronization between the transmitter-and-receiver pair, which accomplishes an initial timing acquisition, is also assumed, as described in Sect. 2.3. 


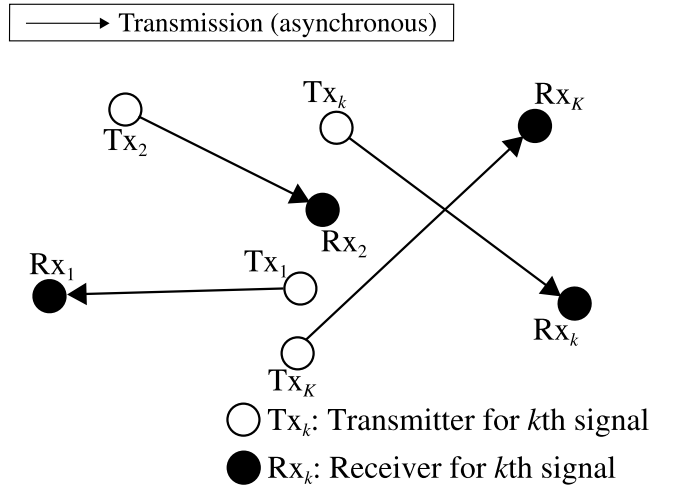

Fig. 1 Decentralized multiple access (no BS and asynchronous transmission).

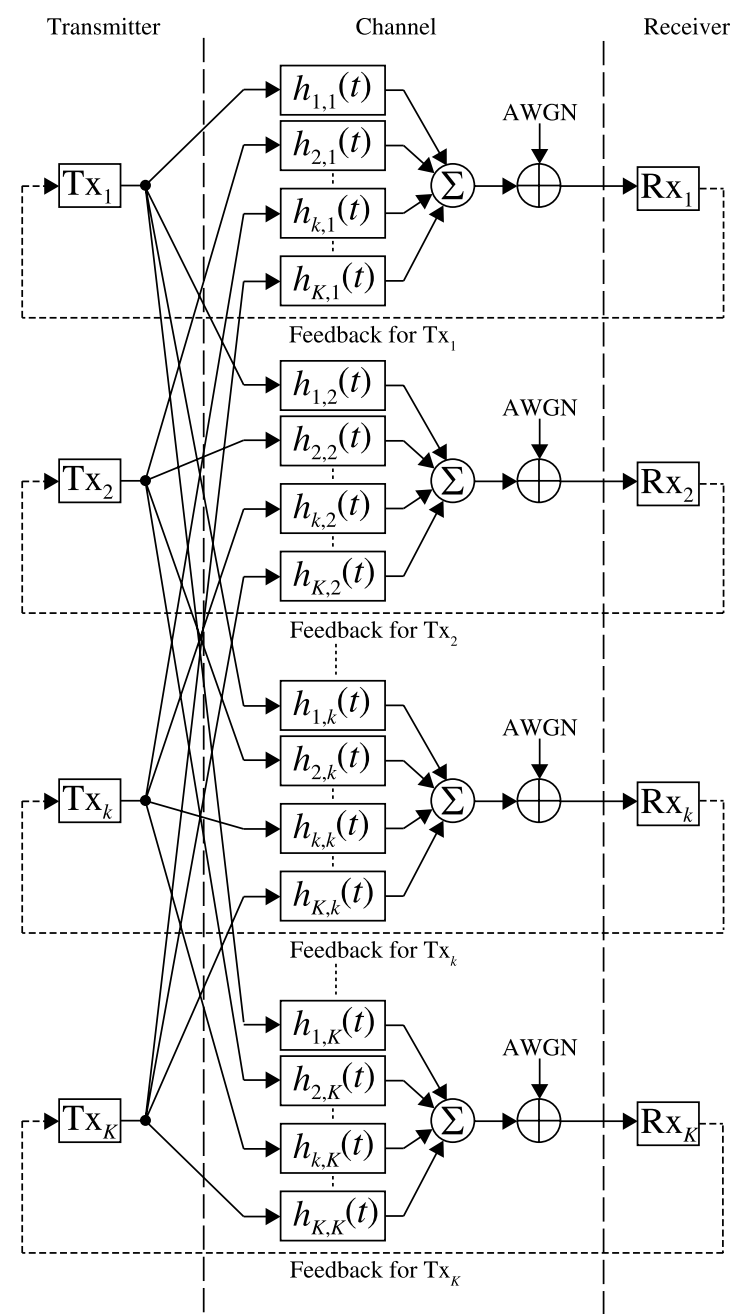

Fig. 2 Asynchronous, decentralized feedback-based system.

receiver for the $k$ th signal of the proposed system are shown in Fig. 3. by

The signature waveform $c_{k}(t)$ for the $k$ th signal is given

$$
c_{k}(t)=\sum_{l=1}^{L} a_{k, l}\left(t-(l-1) T_{c}\right),
$$

where $a_{k, l}(t)\left(0<t<T_{c} ; T_{c}[s]\right.$ is the chip duration $)$ is the $l$ th chip waveform $(l=1,2, \cdots, L)$ for $c_{k}(t)$, given by

$$
a_{k, l}(t)=g(t) \sum_{m=1}^{M} p_{k, l, m} e^{j 2 \pi \xi_{m} t},
$$

where $j=\sqrt{-1}, p_{k, l, m}$ is the complex amplitude of the $m$ th tone of frequency $\xi_{m}[\mathrm{~Hz}](m=1,2, \cdots, M)$ for the $l$ th chip of $c_{k}(t)$. In this paper, we choose $\xi_{m}=\frac{m-1}{T_{c}}$. In the above Eq. (2), $g(t)$ is a rectangular function defined as

$$
g(t)= \begin{cases}1 & \left(0<t<T_{c}\right) \\ 0 & \text { (otherwise) } .\end{cases}
$$

Let $\mathbf{P}_{k}$ be an $L \times M$ matrix that contains $p_{k, l, m}$ such that

$$
\mathbf{P}_{k}=\left[\begin{array}{cccc}
p_{k, 1,1} & p_{k, 1,2} & \cdots & p_{k, 1, M} \\
p_{k, 2,1} & p_{k, 2,2} & \cdots & p_{k, 2, M} \\
\vdots & \vdots & \ddots & \vdots \\
p_{k, L, 1} & p_{k, L, 2} & \cdots & p_{k, L, M}
\end{array}\right] .
$$

The matrix $\mathbf{P}_{k}$ is the hopping pattern for the $k$ th signal.

The $k$ th signal transmitted by the transmitter $\mathrm{Tx}_{k}$ is given by

$$
s_{k}(t)=\sum_{n=0}^{\infty} d_{k}(n) c_{k}\left(t-n T_{s}\right),
$$

where $d_{k}(n)=b_{k}(n) d_{k}(n-1)$ is a differentially encoded complex symbol transmitted in $n T_{s}<t<(n+1) T_{s}(n=$ $0,1, \cdots), b_{k}(n)$ is a complex message symbol, and $T_{s}[\mathrm{~s}]$ is the symbol duration $\left(T_{s}=L T_{c}\right)$. In this paper, we assume that $b_{k}(n)$ is a quaternary phase-shift keying (QPSK) symbol.

\subsection{Channel}

Let $h_{k^{\prime}, k}(t)$ be the impulse response of the channel through which the $k^{\prime}$ th signal $\left(k^{\prime}=1,2, \cdots, K\right)$ is transmitted from $\mathrm{Tx}_{k^{\prime}}$ to the $k$ th receiver $\mathrm{Rx}_{k}$, given by

$$
h_{k^{\prime}, k}(t)=\sum_{i=1}^{I_{k^{\prime}, k}} h_{k^{\prime}, k, i} \delta\left(t-\tau_{k^{\prime}, k, i}\right),
$$

where $h_{k^{\prime}, k, i}$ is the complex gain constant for the $i$ th path of the channel, $\tau_{k^{\prime}, k, i}\left(0 \leq \tau_{k^{\prime}, k, i}<T_{s}\right)$ is the delay for the $i$ th path, and $I_{k^{\prime}, k}$ is the number of paths of the channel.

The received signal $r_{k}(t)$ at the position of the $k$ th receiver is given by

$$
\begin{aligned}
r_{k}(t) & =\sum_{k^{\prime}=1}^{K}\left(s_{k^{\prime}}(t) * h_{k^{\prime}, k}(t)\right)+n(t) \\
& =\sum_{k^{\prime}=1}^{K} \sum_{n=0}^{\infty} \sum_{i=1}^{I_{k^{\prime}, k}} h_{k^{\prime}, k, i} d_{k}(n) c_{k}\left(t-n T_{s}-\tau_{k^{\prime}, k, i}\right)+n(t),
\end{aligned}
$$




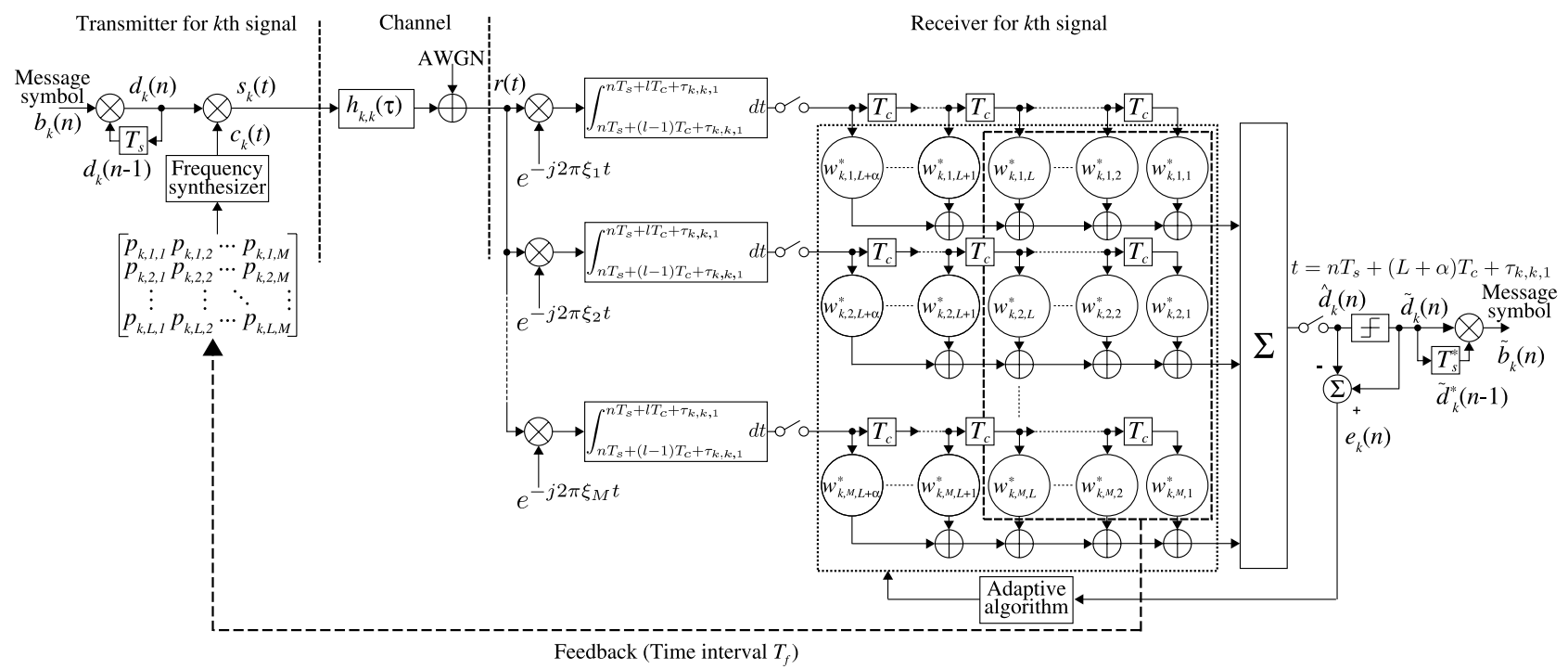

Fig. 3 Transmitter and receiver for the $k$ th signal.

where $n(t)$ is an additive white Gaussian noise (AWGN) with a double-sided power spectral density of $N_{0} / 2$ [W/Hz].

\subsection{Receiver}

The receiver is composed of the adaptive FIR filter, which has $(L+\alpha) \times M$ complex weights $(0 \leq \alpha \leq L)$. Let $\mathbf{W}_{k}$ be an $(L+\alpha) \times M$ matrix that contains the complex weights $w_{k, l, m}$ of the $k$ th receiver.

$$
\mathbf{W}_{k}=\left[\begin{array}{cccc}
w_{k, 1,1} & w_{k, 1,2} & \cdots & w_{k, 1, M} \\
w_{k, 2,1} & w_{k, 2,2} & \cdots & w_{k, 2, M} \\
\vdots & \vdots & \ddots & \vdots \\
w_{k, L, 1} & w_{k, L, 2} & \cdots & w_{k, L, M} \\
w_{k, L+1,1} & w_{k, L+1,2} & \cdots & w_{k, L+1, M} \\
w_{k, L+2,1} & w_{k, L+2,2} & \cdots & w_{k, L+2, M} \\
\vdots & \vdots & \ddots & \vdots \\
w_{k, L+\alpha, 1} & w_{k, L+\alpha, 2} & \cdots & w_{k, L+\alpha, M}
\end{array}\right]
$$

The weight matrix $\mathbf{W}_{k}$ is updated by an adaptive algorithm. In this paper, we adopt a normalized least-mean-square $(\mathrm{N}$ LMS) algorithm [6], which is one of less complex adaptation algorithms, as an example.

For simplicity, we assume that the $k$ th receiver is synchronized with the first path of the desired signal $s_{k}(t)$. The $k$ th receiver obtains discrete-time samples of every frequency and chip from the received signal $r_{k}(t)$. The $m$ th frequency component $r_{k, l, m}$, detected at $t=n T_{s}+l T_{c}+\tau_{k, k, 1}$ $(l=1,2, \cdots, L+\alpha)$, is given by

$$
\begin{aligned}
r_{k, l, m}(n) & =\int_{n T_{s}+(l-1) T_{c}+\tau_{k, k, 1}}^{n T_{s}+l T_{c}+\tau_{k, k, 1}} r_{k}(t) e^{-j 2 \pi \xi_{m} t} d t \\
& =\int_{n T_{s}+(l-1) T_{c}+\tau_{k, k, 1}}^{n T_{s}+l T_{c}+\tau_{k, k, 1}} r_{k}(t) e^{-j \frac{2 \pi(m-1)}{T_{c}} t} d t .
\end{aligned}
$$

We define an $(L+\alpha) \times M$ matrix $\mathbf{R}_{k}(n)$ that contains the samples detected in $n T_{s}+\tau_{k, k, 1}<t<n T_{s}+(L+\alpha) T_{c}+\tau_{k, k, 1}$ as follows:

$$
\begin{aligned}
& \mathbf{R}_{k}(n)= \\
& {\left[\begin{array}{cccc}
r_{k, 1,1}(n) & r_{k, 1,2}(n) & \cdots & r_{k, 1, M}(n) \\
r_{k, 2,1}(n) & r_{k, 2,2}(n) & \cdots & r_{k, 2, M}(n) \\
\vdots & \vdots & \ddots & \vdots \\
r_{k, L, 1}(n) & r_{k, L, 2}(n) & \cdots & r_{k, L, M}(n) \\
r_{k, 1,1}(n+1) & r_{k, 1,2}(n+1) & \cdots & r_{k, 1, M}(n+1) \\
r_{k, 2,1}(n+1) & r_{k, 2,2}(n+1) & \cdots & r_{k, 2, M}(n+1) \\
\vdots & \vdots & \ddots & \vdots \\
r_{k, \alpha, 1}(n+1) & r_{k, \alpha, 2}(n+1) & \cdots & r_{k, \alpha, M}(n+1)
\end{array}\right] .}
\end{aligned}
$$

In this paper, the matrix $\mathbf{R}_{k}(n)$ is called the received signal matrix for the $k$ th receiver.

The FIR filter output $\hat{d}_{k}(n)$ can be represented as

$$
\hat{d}_{k}(n)=\operatorname{tr}\left[\mathbf{W}_{k}^{H}(n) \mathbf{R}_{k}(n)\right],
$$

where the superscript ${ }^{H}$ denotes the complex conjugate and transpose of the matrix, and $\operatorname{tr}[\cdot]$ denotes the trace of the matrix. To recover the message symbol $b_{k}(n)$, the receiver determines the sign for the real and imaginary parts of $\hat{d}_{k}(n)$, such that

$$
\tilde{d}_{k}(n)=\operatorname{sgn}\left[\operatorname{Re}\left[\hat{d}_{k}(n)\right]\right]+j \operatorname{sgn}\left[\operatorname{Im}\left[\hat{d}_{k}(n)\right]\right],
$$

where $\operatorname{sgn}[\cdot]$ is the signum function, $\operatorname{Re}[\cdot]$ is the real part of the complex value, and $\operatorname{Im}[\cdot]$ is the imaginary part of the complex value. Using $\tilde{d}_{k}(n)$, the estimate $\tilde{b}_{k}(n)$ of the complex message symbol $b_{k}(n)$ is given by

$$
\begin{aligned}
\tilde{b}_{k}(n)= & \tilde{d}_{k}(n) \tilde{d}_{k}^{*}(n-1) \\
= & \left(\operatorname{sgn}\left[\operatorname{Re}\left[\hat{d}_{k}(n)\right]\right]+j \operatorname{sgn}\left[\operatorname{Im}\left[\hat{d}_{k}(n)\right]\right]\right) \\
& \times\left(\operatorname{sgn}\left[\operatorname{Re}\left[\hat{d}_{k}(n-1)\right]\right]-j \operatorname{sgn}\left[\operatorname{Im}\left[\hat{d}_{k}(n-1)\right]\right]\right),
\end{aligned}
$$


where the superscript * denotes the complex conjugate.

The weight matrix $\mathbf{W}_{k}(n)$ is updated as follows:

$$
\mathbf{W}_{k}(n+1)=\mathbf{W}_{k}(n)+\frac{\mu}{\left\|\mathbf{R}_{k}(n)\right\|_{\mathrm{F}}^{2}} \mathbf{R}_{k}(n) e_{k}^{*}(n),
$$

where $\mu$ is the step size parameter, $\left\|\mathbf{R}_{k}(n)\right\|_{\mathrm{F}}$ denotes the Frobenius norm of the received signal matrix $\mathbf{R}_{k}(n)$ defined as

$$
\left\|\mathbf{R}_{k}(n)\right\|_{\mathrm{F}}=\sqrt{\sum_{l=1}^{L+\alpha} \sum_{m=1}^{M}\left|r_{k, l, m}(n)\right|^{2}},
$$

and $e_{k}(n)$ is

$$
e_{k}(n)=\tilde{d}_{k}(n)-\operatorname{tr}\left[\mathbf{W}_{k}^{H}(n) \mathbf{R}_{k}(n)\right] .
$$

In this paper, the initial value $\mathbf{W}_{k}(0)$ of the weight matrix $\mathbf{W}_{k}(n)$ for the $k$ th receiver is chosen to be a set of weights that consists of the corresponding initial hopping pattern $\mathbf{P}_{k}(0)$ and the zero matrix $\mathbf{0}_{\alpha \times M}$ of size $\alpha \times M$, that is,

$$
\mathbf{W}_{k}(0)=\left[\begin{array}{lll}
\mathbf{P}_{k}^{T}(0) & \mathbf{0}_{\alpha \times M}^{T}
\end{array}\right]^{T},
$$

where the superscript ${ }^{T}$ denotes the transpose of the matrix.

\subsection{Feedback}

Part of the FIR filter weights of the $k$ th receiver are fed back to the corresponding $k$ th transmitter, in which they are used as an updated version of the hopping pattern $\mathbf{P}_{k}$. In this paper, no delay time and no error for the feedback are assumed ${ }^{\dagger}$. Therefore, the hopping pattern $\mathbf{P}_{k}(\lambda)$ updated at $t=\lambda T_{f}+\Delta_{k}+\alpha T_{c}+\tau_{k, k, 1}\left(\lambda=1,2, \cdots, N_{f} ; N_{f}\right.$ is the number of iterations of the feedback, $T_{f}$ is the feedback time interval, $\Delta_{k}$ is the preassigned offset of the feedback timing $\left.\left(0 \leq \Delta_{k}<T_{f}\right)\right)$ is represented as

$$
\begin{array}{r}
\mathbf{P}_{k}(\lambda)\left(\triangleq\left[\begin{array}{cccc}
p_{k, 1,1} & p_{k, 1,2} & \cdots & p_{k, 1, M} \\
p_{k, 2,1} & p_{k, 2,2} & \cdots & p_{k, 2, M} \\
\vdots & \vdots & \ddots & \vdots \\
p_{k, L, 1} & p_{k, L, 2} & \cdots & p_{k, L, M}
\end{array}\right]\right) \\
=\left[\begin{array}{cccc}
w_{k, 1,1}\left(\hat{n}_{k}\right) & w_{k, 1,2}\left(\hat{n}_{k}\right) & \cdots & w_{k, 1, M}\left(\hat{n}_{k}\right) \\
w_{k, 2,1}\left(\hat{n}_{k}\right) & w_{k, 2,2}\left(\hat{n}_{k}\right) & \cdots & w_{k, 2, M}\left(\hat{n}_{k}\right) \\
\vdots & \vdots & \ddots & \vdots \\
w_{k, L, 1}\left(\hat{n}_{k}\right) & w_{k, L, 2}\left(\hat{n}_{k}\right) & \cdots & w_{k, L, M}\left(\hat{n}_{k}\right)
\end{array}\right],
\end{array}
$$

where $\hat{n}_{k} \triangleq\left\lfloor\left(\lambda T_{f}+\Delta_{k}+\alpha T_{c}+\tau_{k, k, 1}\right) / T_{s}\right\rfloor(\lfloor q\rfloor$ is the maximum positive integer less than or equal to $q$ ). Note that the hopping patten $\mathbf{P}_{k}(\lambda)$ given by (22) corresponds to the upper $L$ rows of the weight matrix $\mathbf{W}_{k}$ given by (9). In this paper, $\Delta_{k}(k=1,2, \cdots, K)$ are chosen to be independent and uniformly distributed random variables in $\left[0, T_{f}\right)$. The FIR filter receiver produces the filter weights that are the MMSE solution to the reference $\tilde{d}_{k}(n)$. As a result, the FIR filter receiver obtains the minimum ISI and MAI for the present received signals. The feedback changes the transmitting signal, and then the FIR filter receiver produces the other filter weights that are another MMSE solution to the reference $\tilde{d}_{k}(n)$. By repeating the feedback, the converged hopping pattern achieves a small BER.

It is known that the RAKE receiver is a receiver that detects the sum of the outputs of a correlator bank, which has time-shifted, weighted despreading sequences ${ }^{\dagger \dagger}$. Therefore, the RAKE receiver can basically be represented by an equivalent single correlator using the sum-vector of the above-mentioned sequences (the time-shifted, weighted despreading sequences). The weights of the proposed receiver are similar to such a sum-vector of despreading sequences. Since the proposed receiver itself behaves like a RAKE receiver, the proposed system does not require explicit RAKE combining. The main difference between them is that the proposed receiver works to produce weights that obtain the highest SINR, whereas the RAKE receiver basically works to obtain the highest signal-to-noise ratio (SNR) using the MRC. This means that the RAKE receiver collects the desired signal's components to enhance SNR, but also collects both the MAI and ISI components, which significantly influence the total SINR. Furthermore, the proposed system contains a feedback mechanism, which changes the transmitted signal, whereby the received signal is changed into an inherently better signal, which results in small MAI and ISI components at the receiver filter output, in a self-organized fashion.

\section{Performance Evaluation}

\subsection{Specifications}

\subsubsection{Initial Hopping Pattern}

We define the initial hopping pattern $\mathbf{P}_{k}(0)$ of $L=7$ and $M=8$ using an FH code, proposed by Einarsson [15], and $M$ Gold sequences of length $L$. The FH code $\mathbf{y}_{k}$ is given by

$$
\begin{aligned}
\mathbf{y}_{k} & =x_{k} \cdot \boldsymbol{\beta} \oplus \gamma_{k} \cdot \mathbf{1} \\
& =\left[\begin{array}{llll}
y_{k, 1} & y_{k, 2} & \cdots & y_{k, L}
\end{array}\right]^{T},
\end{aligned}
$$

where $\boldsymbol{\beta}=\left[\begin{array}{llll}\beta^{0} & \beta^{1} & \cdots & \beta^{L-1}\end{array}\right]^{T}, \beta$ is a primitive element of $\mathrm{GF}\left(M=2^{3}\right), x_{k}, \gamma_{k} \in \mathrm{GF}\left(2^{3}\right), 1$ is the all-one column vector of length $L$, and $\oplus$ and $\cdot$ are addition and multiplication over $\mathrm{GF}\left(2^{3}\right)$, respectively. The values of $x_{k}$ and $\gamma_{k}$ for the $k$ th signal are chosen such that $(k-1)=\gamma_{k} M+x_{k}$. Let $\mathbf{V}_{k}$ be an $L \times M$ matrix whose $(l, m)$ th element $v_{k, l, m}$ is

$$
v_{k, l, m}=\left\{\begin{array}{ll}
1 & \left(m=y_{k, l}+1\right) \\
0 & \left(m \neq y_{k, l}+1\right)
\end{array} .\right.
$$

†The assumption "no delay time (for the feedback)" is irrelevant to the BER performance under the condition that the channel is time-invariant as we assume, and the assumption "no error for the feedback" often requires techniques such as error control coding and automatic repeat request (ARQ) in realistic applications.

$\dagger$ Typically, the maximal-ratio combining (MRC) weights are employed. 
We define the set of $M$ diagonal matrices $\mathbf{Z}_{0}, \mathbf{Z}_{1}, \cdots, \mathbf{Z}_{M-1}$, each of which contains one of the $M$ Gold sequences in its main diagonal. The initial hopping pattern $\mathbf{P}_{k}(0)$ for the $k$ th signal is expressed using $\mathbf{Z}_{x_{k}}$ and $\mathbf{V}_{k}$ as follows:

$$
\mathbf{P}_{k}(0)=\mathbf{Z}_{x_{k}} \mathbf{V}_{k}
$$

Example (for $k=2)$ :

$$
\begin{aligned}
& \mathbf{y}_{2}=\left[\begin{array}{lllllll}
1 & 2 & 4 & 3 & 6 & 7 & 5
\end{array}\right]^{T} \\
& \mathbf{V}_{2}=\left[\begin{array}{llllllll}
0 & 1 & 0 & 0 & 0 & 0 & 0 & 0 \\
0 & 0 & 1 & 0 & 0 & 0 & 0 & 0 \\
0 & 0 & 0 & 0 & 1 & 0 & 0 & 0 \\
0 & 0 & 0 & 1 & 0 & 0 & 0 & 0 \\
0 & 0 & 0 & 0 & 0 & 0 & 1 & 0 \\
0 & 0 & 0 & 0 & 0 & 0 & 0 & 1 \\
0 & 0 & 0 & 0 & 0 & 1 & 0 & 0
\end{array}\right] \\
& \mathbf{Z}_{x_{2}=1}=\left[\begin{array}{ccccccc}
+ & 0 & 0 & 0 & 0 & 0 & 0 \\
0 & + & 0 & 0 & 0 & 0 & 0 \\
0 & 0 & - & 0 & 0 & 0 & 0 \\
0 & 0 & 0 & + & 0 & 0 & 0 \\
0 & 0 & 0 & 0 & + & 0 & 0 \\
0 & 0 & 0 & 0 & 0 & + & 0 \\
0 & 0 & 0 & 0 & 0 & 0 & -
\end{array}\right] \\
& \mathbf{P}_{2}(0)=\mathbf{Z}_{x_{2}=1} \mathbf{V}_{2} \\
& =\left[\begin{array}{cccccccc}
0 & + & 0 & 0 & 0 & 0 & 0 & 0 \\
0 & 0 & + & 0 & 0 & 0 & 0 & 0 \\
0 & 0 & 0 & 0 & - & 0 & 0 & 0 \\
0 & 0 & 0 & + & 0 & 0 & 0 & 0 \\
0 & 0 & 0 & 0 & 0 & 0 & + & 0 \\
0 & 0 & 0 & 0 & 0 & 0 & 0 & + \\
0 & 0 & 0 & 0 & 0 & - & 0 & 0
\end{array}\right]
\end{aligned}
$$

\subsubsection{Multipath Model}

To evaluate the performance of the proposed system, we assume a six-path model (i.e., $I_{k^{\prime}, k}=6$ for all indices of $k^{\prime}$ and $k$ ) that has a delay profile of exponential decay, as shown in Fig. 4, where the relative intensities of $\left|h_{k^{\prime}, k, i}\right|$ are $20 \log _{10}\left(\left|h_{k^{\prime}, k, i+1}\right| /\left|h_{k^{\prime}, k, i}\right|\right)=-3 \mathrm{~dB}\left(i=1,2, \cdots, I_{k^{\prime}, k}-1\right)$, the path delays $\tau_{k^{\prime}, k, i}$ are $\tau_{k^{\prime}, k, i+1}-\tau_{k^{\prime}, k, i}=\frac{L+1}{16} T_{c}\left(\approx \frac{1}{16} T_{s}\right.$

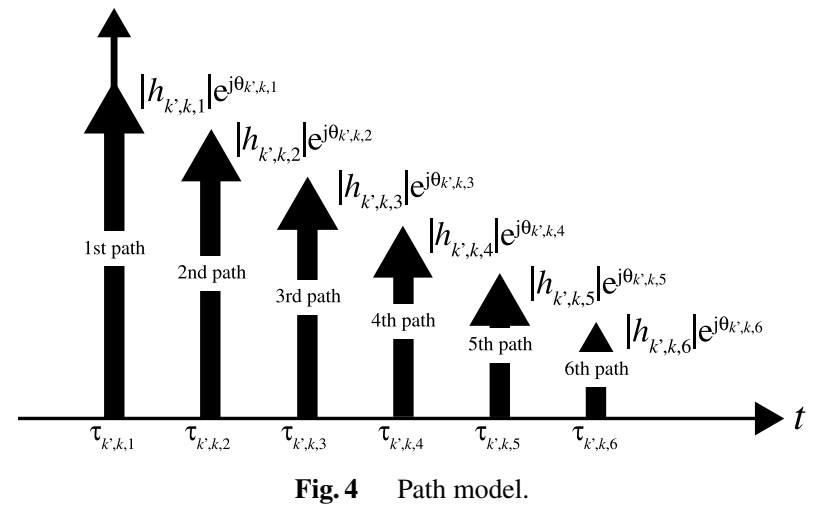

for $L=7$ ), and $\tau_{k^{\prime}, k, 1}$ (for all $k^{\prime}$ and $k$ ) and $\theta_{k^{\prime}, k, i}$ (for all $k^{\prime}$, $k$, and $i$ ) are mutually statistically independent, uniformly distributed random variables in the intervals of $\left[0, T_{s}\right)$ and $[0,2 \pi)$, respectively. Note that although we assume an identical amplitude decay of $-3 \mathrm{~dB}$ for all $k^{\prime}$ and $k$ for simplicity, $\tau_{k^{\prime}, k, 1}$ and $\theta_{k^{\prime}, k, i}$ are chosen to be independent for all $k^{\prime}, k$, and $i$. This causes extremely severe channel conditions for multiple access.

\subsubsection{Other Specifications}

The proposed system requires an initial training period during which the receiver returns part of the filter weights to the corresponding transmitter to construct a suitable hopping pattern for the current channel state. In this paper, we define the initial training period as $t<\left(N_{f}+1\right) T_{f}+\Delta_{k}+\tau_{k, k, 1}$, and discuss the BER performance in the steady period, which is defined as the period after the initial training period, that is, $t \geq\left(N_{f}+1\right) T_{f}+\Delta_{k}+\tau_{k, k, 1}$. In the steady period, only the updating of filter weights is performed at the receiver (i.e., no feedback). We assume that the reference $\tilde{d}_{k}(n)$ used for the update of the filter weights is $\tilde{d}_{k}(n)=d_{k}(n)$ during the initial training period, which implies that the receiver has prior knowledge of the pilot data symbols used for the initial training. The demodulated $\tilde{d}_{k}(n)$ can also be used for the update of the filter weights such as in [14], so that the overhead (or pilot data symbols) for running the proposed system becomes small. Since the BER performance slightly depends on the randomly chosen values of $\tau_{k^{\prime}, k, 1}$ and $\theta_{k^{\prime}, k, i}$, all plots show the average BER of five simulation trials. Other common specifications are listed in Table 1.

\subsection{Simulation Results}

\subsubsection{Decentralized Multiple-Access Performance}

We evaluate the BER performance of the proposed system in comparison with the conventional DS-CDMA using Gold sequences with the matched filter (MF) with/without the RAKE combining method (six-finger maximal-ratio combining), and the FCSS/DS-CDMA ${ }^{\dagger}$.

Figure 5 shows the characteristics of BER vs. the number of active signals $K$. It can be seen from Fig. 5 that the BER of the conventional DS-CDMA rapidly increases with the number of active signals $K$, even though the RAKE combining is employed, because the RAKE receiver collects the MAI and ISI components in addition to the desired signal's components, as was elaborated in Sect.2.4. On the other hand, the degradation of the BER of the proposed system $\left(\alpha=7, N_{f}=10\right)$ is the most gradual with the increase in $K$. This indicates that the feedback mechanism of the proposed system, which yields inherently better received signal for each receiver as was elaborated in Sect. 2.4, effectively

†Throughput is also an important figure. The main factor that influences the throughput in the proposed system is the required number of data bits for the feedback, which is discussed in Sect. 3.2. 
Table 1 Common specifications.

\begin{tabular}{|c|c|c|c|}
\hline & Proposed system & FCSS/DS-CDMA & DS-CDMA (MF, RAKE) \\
\hline Data & \multicolumn{3}{|c|}{ Differentially encoded QPSK } \\
\hline$E_{b} / N_{0}$ & \multicolumn{3}{|c|}{$9.9 \mathrm{~dB}$} \\
\hline$L$ & 7 & \multicolumn{2}{|r|}{31} \\
\hline$\alpha$ & 0,7 & 0,31 & - \\
\hline$M$ & 8 & \multicolumn{2}{|r|}{1} \\
\hline$T_{f}$ & \multicolumn{2}{|c|}{$10^{4} T_{s}$} & - \\
\hline$N_{f}$ & \multicolumn{2}{|c|}{0,10} & - \\
\hline$\Delta_{k}$ & \multicolumn{2}{|c|}{$\begin{array}{c}\text { Uniform distribution in }\left[0, T_{f}\right) \\
\text { N-LMS }(\mu=01)\end{array}$} & - \\
\hline Adaptive algorithm & $\mathrm{N}-\mathrm{LM}$ & $(\mu=0.1)$ & - \\
\hline
\end{tabular}

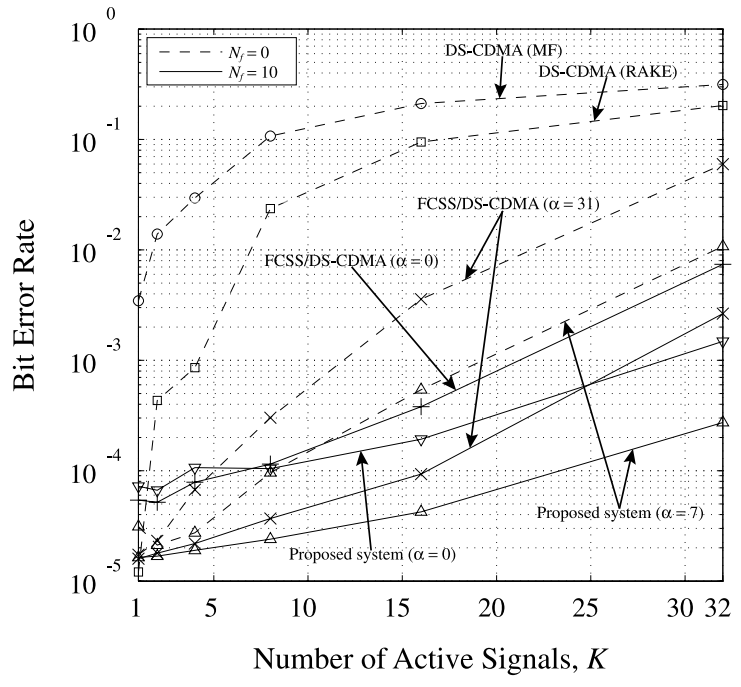

Fig. 5 Asynchronous, decentralized multiple-access performance (proposed system $\left(\alpha=0,7, N_{f}=0,10, T_{f}=10^{4} T_{s}\right)$ vs. several conventional systems).

works and improves the total SINR at all the receivers in a self-organized fashion. As a result, significant improvement in BER can be obtained, particularly for a large number of active signals, as shown in Fig. 5.

Figure 6 shows the characteristics of BER vs. $E_{b} / N_{0}$ for $K=32$. It can be observed that the proposed system $\left(\alpha=7, N_{f}=10\right)$ results in a $3 \mathrm{~dB}$ gain at BER $=10^{-3}$ relative to the FCSS/DS-CDMA ( $\left.\alpha=31, N_{f}=10\right)$. To discuss the effectiveness of the proposed system for limited feedback channels, we consider the influence of quantization by which the required number of data bits for the feedback is changed. We assume a uniform quantization [14]. Let $\rho$ be the number of quantization bits per real or imaginary part per chip per frequency. Therefore, the total number of data bits required for one feedback can be represented as $2 \rho L M$. It is observed from Fig. 6 that $\rho=6$ is sufficient to achieve good BER performance comparable to $\rho=\infty$. This means that the proposed system is effective for limited feedback channels.

Examples of the initial hopping pattern, the converged hopping pattern, and their power spectra are shown in Fig. 7, where tone levels $p_{k, l, m}$ are represented by their absolute values $\left|p_{k, l, m}\right|$. Although every chip of the initial hopping pattern contains a single tone, it can be seen from Fig. 7(c) that

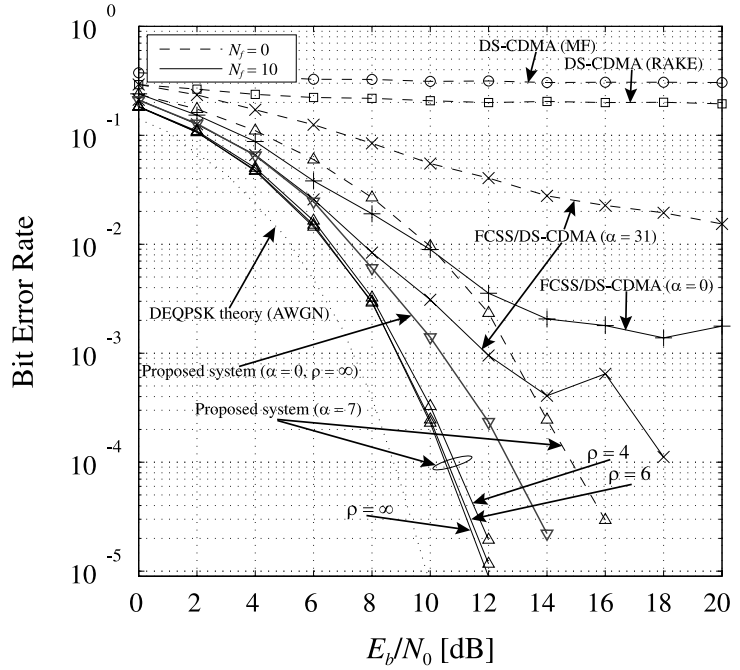

Fig. 6 BER characteristic for $K=32\left(\alpha=0,7, N_{f}=0,10, T_{f}=\right.$ $\left.10^{4} T_{s}\right)$.

the converged hopping pattern contains multiple tones.

\subsubsection{Uplink Performance}

Here, we assume uplink multiple access. This can be regarded as a special case of the decentralized multiple access in which all the receivers are positioned at the same location such as at a BS. In other words, the impulse response functions $h_{k^{\prime}, k}(t)$, given by (6), are identical for all $k$ $(k=1,2, \cdots, K)$, that is, $h_{k^{\prime}, 1}(t)=h_{k^{\prime}, 2}(t)=\cdots=h_{k^{\prime}, K}(t) \triangleq$ $\left.h_{k^{\prime}}(t)\right)$, as shown in Fig. 8 . Note that for asynchronous, decentralized multiple access, $K^{2}$ impulse responses were needed to be considered in a system, as was shown in Fig. 2 . On the other hand, only $K$ impulse responses are needed for asynchronous uplink multiple access. Therefore, since a fewer number of unknown variables implies a better convergence of a system in general, it is expected that the asynchronous uplink multiple-access performance of the proposed system is better than the asynchronous, decentralized multiple-access performance.

Figure 9 shows the uplink BER performance as a function of the number of active signals. It can be observed that the proposed system gives an excellent uplink performance. 


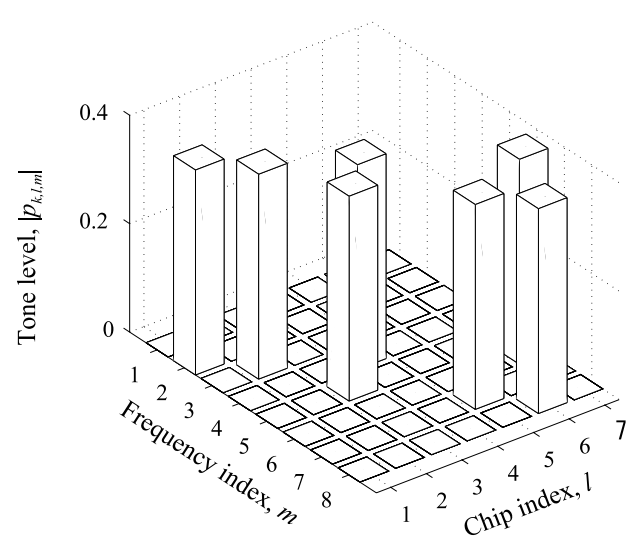

(a) Initial hopping pattern.

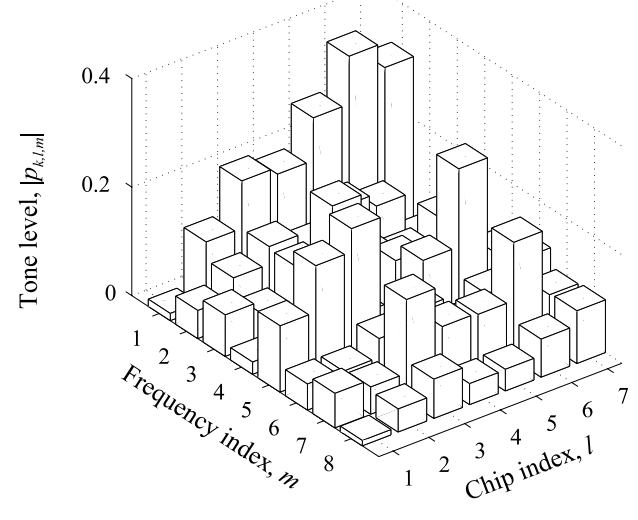

(c) Converged hopping pattern.

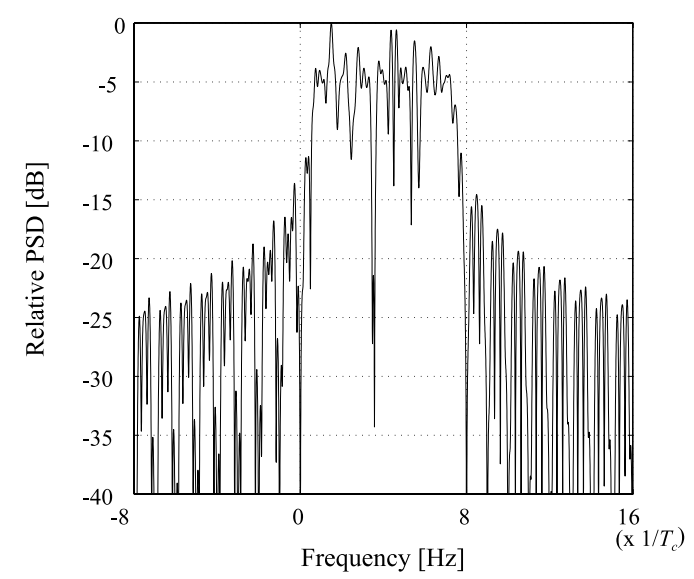

(b) Power spectrum of initial hopping pattern.

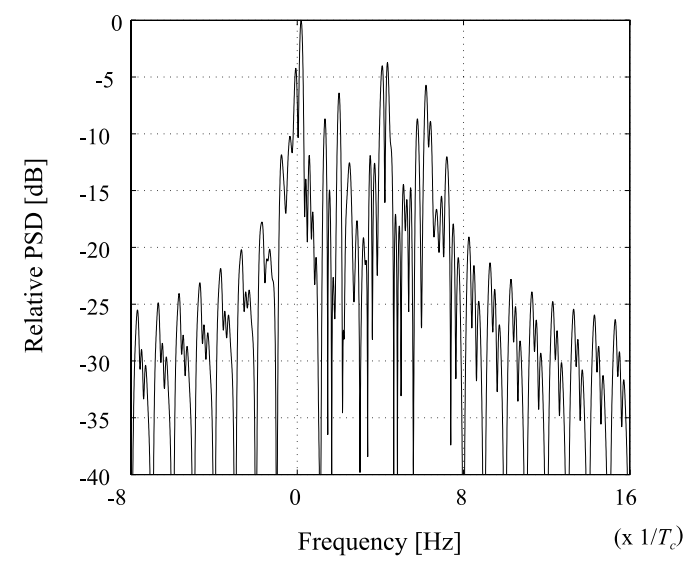

(d) Power spectrum of converged hopping pattern.

Fig. 7 Example of a converged hopping pattern and its power spectrum.

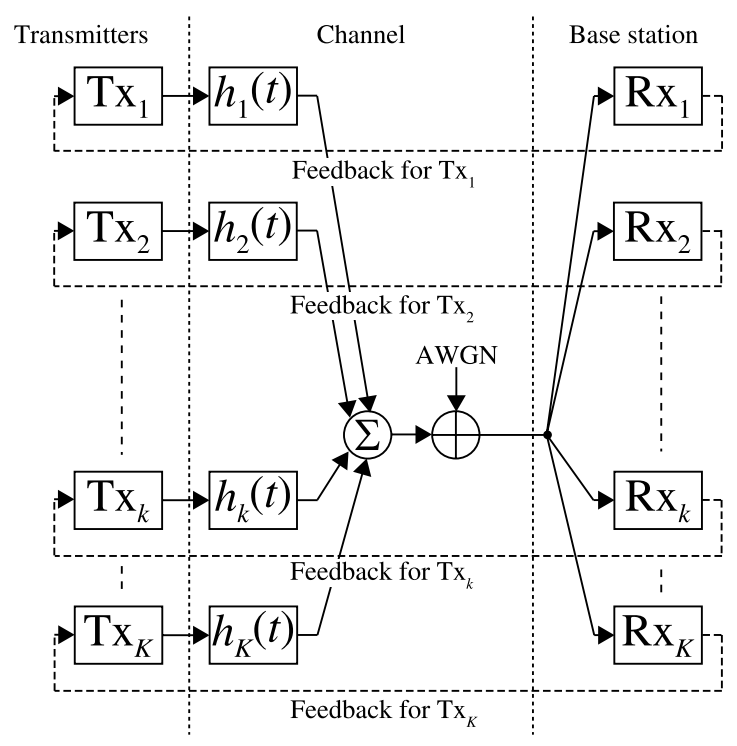

Fig. 8 Uplink multiple access.

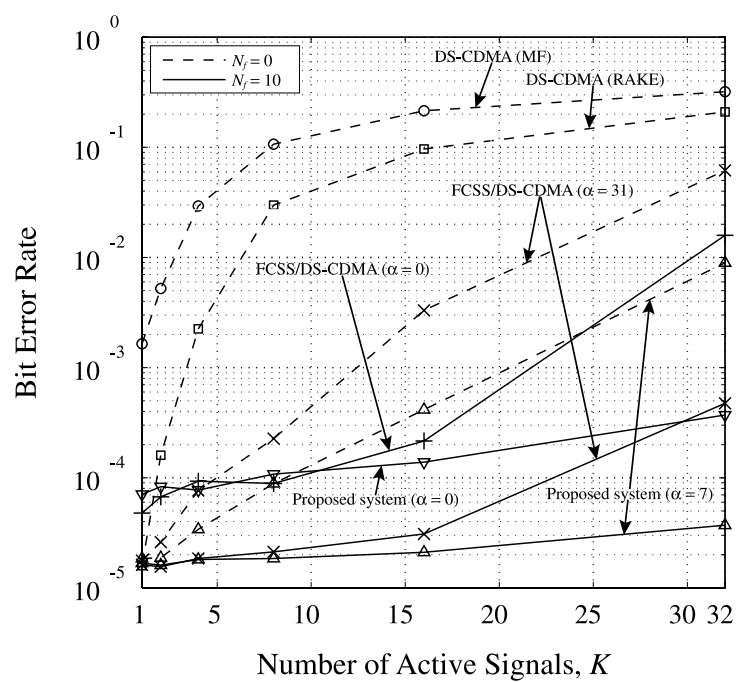

Fig. 9 Asynchronous uplink performance (proposed system ( $\alpha=0,7$, $\left.N_{f}=0,10, T_{f}=10^{4} T_{s}\right)$ vs. several conventional systems). 


\section{Conclusion}

In this paper, an FCHP/MH-CDMA was proposed, and decentralized multiple-access performance and uplink performance were evaluated. It has been shown that the FCHP/MH-CDMA provides us with an excellent BER performance. The FCHP/MH-CDMA is a promising candidate for spectrum-agile technologies.

\section{Acknowledgment}

This work was supported in part by a Grant-in-Aid for Young Scientists (B19760271) from the Ministry of Education, Culture, Sports, Science and Technology (MEXT) in Japan.

\section{References}

[1] J.G. Proakis, Digital Communications, 3rd ed., McGraw-Hill, New York, 1995.

[2] W.C. Jakes, Microwave Mobile Communications, IEEE Press, New Jersey, 1974.

[3] T.S. Rappaport, Wireless Communications, 2nd ed., Prentice Hall, New Jersey, 2002.

[4] G. Marubayashi, M. Nakagawa, and R. Kohno, Spread Spectrum Communications and Its Applications, IEICE, Tokyo, 1998.

[5] R. Esmailzadeh and M. Nakagawa, "Pre-RAKE diversity combination for direct-sequence spread spectrum mobile communications systems," IEICE Trans. Commun., vol.E76-B, no.8, pp.1008-1015, Aug. 1993.

[6] S. Haykin, Adaptive Filter Theory, 3rd ed., Prentice Hall, New Jersey, 1996.

[7] S. Yoshida, A. Ushirokawa, S. Yanagi, and Y. Furuya, "DS/CDMA adaptive interference canceller on differential detection for fast fading channel," IEICE Trans. Commun. (Japanese Edition), vol.J77B-II, no.11, pp.618-627, Nov. 1994.

[8] U. Madhow and M.L. Honig, "MMSE interference suppression for direct-sequence spread-spectrum CDMA," IEEE Trans. Commun., vol.42, no.12, pp.3178-3188, Dec. 1994.

[9] S. Hamada, M. Hamamura, H. Suzuki, and S. Tachikawa, "A proposed DS/CDMA system using analog PN sequences produced by adaptive filters," IEICE Trans. Fundamentals, vol.E81-A, no.11, pp.2261-2268, Nov. 1998.

[10] S. Ulukus and R.D. Yates, "Iterative constructions of optimum signature sequence sets in synchronous CDMA systems," IEEE Trans. Inf. Theory, vol.47, no.5, pp.1989-1998, July 2001.

[11] L.R. Welch, "Lower bounds on the maximum cross correlation of signals," IEEE Trans. Inf. Theory, vol.20, no.3, pp.397-399, May 1994.

[12] M. Rupf and J.L. Massey, "Optimum sequence multisets for synchronous code-division multiple-access channels,” IEEE Trans. Inf. Theory, vol.40, no.4, pp.1261-1266, July 1994.

[13] T. Miyatake, M. Hamamura, and S. Tachikawa, "Performance of DS/SS system using feedback controlled spreading sequence over a multipath channel," Proc. ISITA2004, pp.567-571, Parma, Oct. 2004.

[14] T. Miyatake, K. Chiba, M. Hamamura, and S. Tachikawa, "Asynchronous, decentralized DS-CDMA using feedback-controlled spreading sequences for time-dispersive channels," IEICE Trans. Commun., vol.E91-B, no.1, pp.53-61, Jan. 2008.

[15] G. Einarsson, "Address assignment for a time-frequency-coded spread-spectrum system," Bell Syst. Tech. J., vol.59, no.7, pp.12411255, Sept. 1980

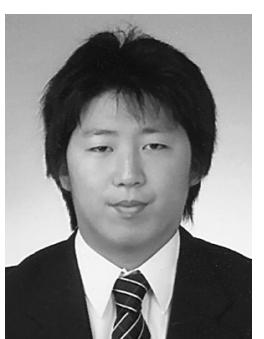

Kazuki Chiba received the B.E. degree in Information Systems Engineering from Kochi University of Technology, Kochi, Japan, in 2008. He is currently working toward the M.E. degree in Information Systems Engineering at Kochi University of Techonology, Kochi, Japan. His research interests lie in the area of feedback-based communication systems.

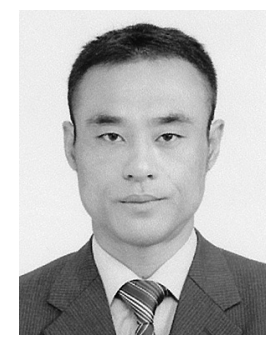

Masanori Hamamura received his B.S. M.S. and Ph.D. degrees in electrical engineering from Nagaoka University of Technology, Nagaoka, Japan, in 1993, 1995 and 1998, respectively. From 1998 to 2000, he was a Research Fellow of the Japan Society for the Promotion of Science. Since 2000, he has been with the Department of Information Systems Engineering at Kochi University of Technology, Kochi, Japan, where he is now an Associate Professor. From 1998 to 1999 , he was a visiting researcher at Centre for Telecommunications Research, King's College London, United Kingdom, where he worked on adaptive signal processing for mobile systems. His current research interests are in the areas of spread spectrum systems, wireless communications and signal processing. Dr. Hamamura is a member of IEEE and SITA of Japan. 